\title{
Site Visit
}

National Cancer Institute

\section{Source}

National Cancer Institute. Site Visit. NCI Thesaurus. Code C19032.

A visit from an oversite group to a site of a study or research for fact gathering, peer review, or compliance with regulations. 